COMUNICACIONES

\title{
PROYECCIÓN DE LA DEMANDA DE MÉDICOS EN MÉXICO 1965-1980
}

\author{
ClaRA J. DE Bialostozky \\ $\mathrm{y}$ \\ DAVID BARKIN \\ El Colegio de México
}

EsTE TRABAJ0* fue presentado por Charles N. Myers al Seminario sobre aplicación de métodos de previsión de la oferta y demanda de recursos humanos de nivel superior, que se llevó a cabo en la ciudad de México en diciembre de 1970. Constituye uno de los pocos ${ }^{1}$ realizados hasta ahora en México para estimar las probables diferencias entre la demanda y la oferta futura de un tipo particular de recursos humanos de alto nivel, en este caso el de los médicos.

Sin embargo, Myers escogió una de las profesiones para las cuales es más difícil prever futuras tendencias, especialmente en lo relativo a la demanda.

El autor elabora un modelo "relativamente simple" utilizando el método de análisis de regresión múltiple con información transversal de las 32 entidades federativas en 1960 y 1965 . Selecciona como variables independientes las siguientes: porciento de la población en áreas urbanas, producto per capita, porciento de la población con 12 años o más de educación, porciento de la población que habita viviendas sin drenaje, porciento de la población que toma regularmente alimentos proteínicos y pan de trigo y porciento de la población que usa regularmente zapatos, para las cuales tiene información de 1960. La variable dependiente es el número de médicos por cada 10000 habitantes en cada entidad en los años 1960 y 1965.

Con base en una serie de limitaciones que señala y después de examinar cada una de las variables, el autor utiliza como el mejor elemento para proyectar la futura demanda de médicos el grado de urbanización de cada una de las entidades federativas. Su medida de urbanización es la censal, es decir, el porciento de población que vive en localidades de 2500 habitantes y más. La ecuación más adecuada para estimar la demanda de médicos, juzgada en términos de su poder

* Véase este mismo número de Demografía Y Economía, pp. 193-227.

1 Otro trabajo de este tipo es el realizado por un grupo de pasantes de la carrera de Ingeniería Química en relación a las perspectivas futuras de dicha carrera, del cual aparecerá una versión resumida en la Revista del centro de Estudios Educativos. 
para explicar la varianza entre el número de médicos y el grado de urbanización, resultó tener una forma no lineal con características de una parábola.

Ahora bien, si tratamos de determinar los factores de los cuales depende realmente la demanda de servicios médicos en México encontramos lo siguiente:

1. Una parte, que creemos es reducida (tal vez un $20 \%$ de la población total), es la demanda ejercida por el estrato de la población que tiene mayor capacidad de pago. Dicha demanda dependerá del nivel de ingresos de la población, es decir, del crecimiento económico del país. Obviamente la parte proporcional de la demanda total que representa este tipo de demanda será mayor en la medida en que aumenten los ingresos de las personas en los estratos más bajos.

2. Otra parte de la demanda, que cubre poco más de un $20 \%$ de la población total, es la ejercida por instituciones tales como el Instituto Mexicano del Seguro Social (IMSs), el Instituto de Seguridad y Servicios Sociales de los Trabajadores del Estado (Issste), Petróleos Mexicanos (PEMEX), Ferrocarriles Nacionales, etc., ${ }^{2}$ que hasta ahora ha estado directamente relacionada con la capacidad del sector moderno de la industria y de los servicios, pero que también depende en forma importante de decisiones de tipo político.

3. Por último, nos encontramos con lo que constituye la llamada demanda social que es la de los sectores que, dados sus bajos niveles de ingresos y el tipo de actividad económica que desarrollan, no participan de ninguna de las dos situaciones señaladas. La satisfacción de esta demanda depende exclusivamente de consideraciones de tipo político. Lo que determina la demanda es el volumen de la oferta y generalmente no existe una contrapartida de parte de los receptores. Se trataría de lo que podríamos llamar beneficencia y entrarían tanto la pública (Secretaría de Salubridad y Asistencia, seguro social al campo, etc.) como la privada (Cruz Roja, servicios médicos dependientes de corporaciones religiosas y organizaciones privadas, etc.).

De lo anterior podemos afirmar que el tratamiento de la demanda de servicios de personal médico en México presenta problemas muy particulares y se aparta en gran medida de los tratamientos tradicionales de demanda de otros bienes y servicios en los que, en general, ésta se hace depender de los ingresos y/o de los precios. Surge la duda de si realmente en este caso particular, "la distribución (o 'demanda') es función de los niveles de urbanización, ingreso, educación y vida, así como de aculturación" ( $p$. 197) y si efectivamente se trata de un servicio que entra en forma íntegra en el juego de la oferta y la demanda del mercado.

El autor del trabajo parece creer en lo anterior y fundamenta su estudio en este tipo de supuestos. Así afirma que: "Para efectuar el análisis es necesario suponer que estas relaciones representan dos conjuntos de puntos de equilibrio entre la demanda del gobierno y la privada sumadas y la oferta total" (p. 195, subrayado nuestro). Afirmar

2 Hay una duplicación entre la población que efectivamente puede ejercer una demanda y la amparada por estas instituciones, por ser precisamente las personas con empleos dentro del sector industrial moderno y el de servicios mejor remunerados. 
que la oferta y la demanda de médicos se encontraba en un punto de equilibrio como hipótesis básica, nos parece un poco fuera de lugar dada la situación actual del país, pues no conlleva un juicio de valor sobre la bondad de la situación. No hay duda que el Estado ha hecho grandes esfuerzos para ampliar el sistema de servicios médicos a través del IMss fundamentalmente, pero las graves deficiencias de ésta son demasiado aparentes, sobre todo la dificultad de satisfacer las demandas más ingentes de la población que vive en zonas donde el servicio aún no ha llegado.

No sería arbitrario afirmar que con excepción de una pequeña proporción de la población, la mayoría de los mexicanos no dispone de los recursos necesarios para ser atendidos por un especialista en medicina. Es por esto que nos parece equivocado hablar de una situación de equilibrio; en este caso, sólo se puede justificar en términos del objetivo principal del autor que es proyectar una "demanda mínima".

Ahora bien, Myers al formular un modelo de regresión lineal con el fin de determinar "únicamente el poder de pronóstico de las variables que parecen estar relacionadas con la demanda" (p. 198), nos parece que incurre en un error de planteamiento que es el de introducir como variables independientes algunas para las cuales resulta demasiado difícil o bien no existen elementos para prever su comportamiento futuro.

Así vemos que después de llegar a determinar que las variables que "explican" mejor las variaciones en el número de médicos por cada 10000 personas en 1965 son el nivel educativo, la urbanización y el consumo de proteínas en 1960, el autor dice: "No se puede utilizar el modelo de rezago al proyectar la demanda dada la dificultad para estimar los valores futuros de las tres variables independientes, en particular el nivel educativo y el consumo de proteínas, respecto a cada entidad, en cada año meta. Los errores que se introdujeran contrarrestarían con exceso el alto poder predictivo de la ecuación. Se tiene que usar en su lugar una ecuación más sencilla con un poder de pronóstico menor, pero basada en una o dos variables independientes que sean relativamente más fáciles de proyectar" (pp. 203, 204 ).

En última instancia, como se dijo antes, el autor utiliza como variable independiente para su proyección el grado de urbanización y toma como medida de éste aquella que define las zonas urbanas a partir de los 2500 habitantes, medida que él mismo reconoce presenta problemas. Aun para este concepto creemos que existen medidas alternativas mejores; por ejemplo, Unikel ha sugerido que una definición a partir de 15000 habitantes daría una aproximación mejor a una definición funcional de un área urbana en México. ${ }^{3}$

En este contexto las conclusiones de Myers pueden reinterpretarse de la siguiente manera: para poder mantener los niveles actuales de servicios médicos, de acuerdo con una población creciente y la pauta de desarrollo urbano que se puede esperar, serán necesarios 50000 médicos adicionales para satisfacer las necesidades en 1980. Myers

3 Luis Unikel, "Ensayo sobre una nueva definición de la población rural y urbana en México", Demografía Y Economía, II: 1 (1968). 
mismo reconoció algunas de las fallas de su metodología al admitir la necesidad de limitar sus estimaciones para no caer por debajo de los niveles actuales de servicio (esto es, el número de médicos por 10000 personas en cada entidad del país en 1960 y 1965); la justificación de lo anterior es el "supuesto de la demanda mínima comprobada" (p. 207). Lo que esto implica es que aun cuando sus ecuaciones sugieren un descenso de la demanda, "probablemente no disminuyó la demanda real" (p. 206).

Otro de los problemas que encontramos en la metodología de Myers es el uso de datos cruzados para proyectar demandas en el tiempo. El autor parece estar consciente del problema metodológico que esto plantea, pero dice que los resultados obtenidos al utilizar la situación de 1960 para predecir la de 1965 confirman la posibilidad del uso de este tipo de dispositivos. Quizás en este caso sea cierto, pero diversos análisis realizados hasta ahora, como son los de la oCDE o un trabajo de uno de los autores ${ }^{4}$ plantean dudas adicionales sobre la validez de dicha metodología.

Creemos que la contribución de Myers al conocimiento de la pro. blemática de la oferta y la demanda de médicos en los años venideros es muy importante. Sus estimaciones, por más gruesas que sean, sugieren un gran desequilibrio en los próximos 15 años; dependiendo de los supuestos usados, éste fluctuaría entre 6000 y 12000 médicos. Nos parece, sin embargo, que la situación es mucho más grave que la sugerida por Myers y que el déficit será, con creces, mucho mayor que el estimado en el trabajo. El aumento necesario calculado por Myers de 15 a $25 \%$ en el número de egresados de escuelas de medicina es, con mucho, una subestimación de las necesidades, dada la persistencia de la tecnología actual. Con esto sólo se llevaría a una perpetuación de la situación presente donde las personas con mayores posibilidades económicas tienen acceso más fácil a los servicios médicos disponibles. No responde a las necesidades "reales" que uno puede suponer manifiestan una relación inversa con el nivel de ingreso familiar.

Sin embargo, pensamos que un tratamiento meramente cuantitativo del problema deja fuera aspectos muy importantes de un problema eminentemente cualitativo. A continuación enunciaremos algunos de éstos.

En primer lugar, nos encontramos con los problemas de la enseñanza médica. Podemos afirmar que en las decisiones que llevan a las personas a ingresar a las escuelas médicas tiene gran influencia el status social de que gozan los médicos, particularmente en las sociedades en desarrollo. Las perspectivas y expectativas de los individuos que estudian medicina son generalmente muy altas. Sumando a esto el efecto demostración de la vida en las zonas urbanas que se hace sentir, por lo menos, durante la carrera de medicina en universidades urbanas, podemos encontrar algunas de las causas de la concentración geográfica de los médicos. La posibilidad de redistribuir geográficamente este tipo de personal es muy remota y se requeriría un cambio importante en los valores sociales.

4 David Barkin, "Acceso a y beneficios de la educación superior", por aparecer en la Revista del Centro de Estudios Educativos. 
A esto se agrega el hecho de que para mantener o mejorar la calidad mínima de la medicina practicada en un país es necesario el trabajo hospitalario, en donde los individuos teinen contacto continuo tanto con profesionales de su propia rama como con los avances científicos en el campo. Los centros hospitalarios están en las áreas urbanas.

Por otro lado, nos encontramos con un alto grado de deserción en las escuelas de medicina y, al varecer, con la obstrucción al flujo de egresados en años recientes. El requisito de internados y residencias se ha convertido en una cuestión de tipo formal ya que no existen suficientes plazas en el país con un nivel mínimo de calidad para poder llevarlos a cabo.

Las alternativas que al parecer se han planteado hasta ahora han sido, entre otras, el intento de reducir el número de años de la carrera, limitar la duración del internado a seis meses y eliminar del curriculum algunas ciencias básicas (fisiología, bioquímica, etc.) o reducir el tiempo destinado a ellas.

En última instancia esto nos lleva al examen de lo que significa calidad de los servicios médicos y creemos que es necesario un replanteamiento fundamental de dicho significado.

La raíz de los problemas de salud pública se encuentra, como es bien sabido y como Myers lo hace notar en su trabajo, en factores ambientales $\mathrm{y}$ nutricionales que van desde los problemas del abastecimiento de agua potable hasta los relativos a deficiencias dietéticas. Del total de viviendas del país, un $39 \%$ no dispone de agua entubada y en un $12 \%$ se obtiene de llave pública o hidrante; en el $21.3,23.8$, $38.9,70.3$ y $24.1 \%$ no se come, respectivamente, carne, huevos, leche, pescado y pan de trigo (en una semana de referencia).$^{5}$ (Esto representa más o menos los mismos porcientos de población.)

El enfrentamiento a estas situaciones depende del reconocimiento del problema como parte de la situación de la salud pública general del país, y requiere de la acción conjunta, coordinada y continua de las diversas dependencias del sector público (Secretaría de Salubridad y Asistencia, Secretaría de Educación Pública, Secretaría de Agricultura y Ganadería, Compañía Nacional de Subsistencias Populares, etc.). Significaría una reestructuración y ampliación del gasto gubernamental en los programas de salud pública.

Además de esto y ya desde el punto de vista específico de los servicios médicos, la alternativa esbozada por Myers en las últimas líneas de su trabajo es muy interesante. La idea seria implementar un sistema de medicina preventiva utilizando personal paramédico. Este personal estaría capacitado para hacer frente a los problemas médicos más importantes en términos del volumen de la población que se ve afectada por ellos, como son las enfermedades gastrointestinales y respiratorias. Este tipo de sistema tendría las siguientes ventajas:

1. Se mejoraría el nivel de salud de la población y se reduciría el volumen de las situaciones críticas a las que tiene que hacer frente la medicina curativa.

5 Dirección General de Estadística, IX Censo General de Población, 1970. Re sumen de las principates características por entidad federativa. México, D. F. 1970. En este censo se hizo un cambio en la forma de obtener esta información que mejoró su validez como indicador estadístico. 
2. Se reorientaría la distribución geográfica de los servicios médicos y como consecuencia se reduciría el costo de aprovechamiento de los servicios de parte de la población.

3. Se contribuiría a un mejoramiento de la distribución del ingreso, pues se afectaría en particular a la población en los estratos bajos.

4. El costo de oferta de servicios paramédicos es menor. Primero por significar un menor costo y número de años de adiestramiento del personal. La complejidad de las instalaciones necesarias es también menor $\mathrm{y}$, por último, la remuneración de este tipo de personal sería relativamente inferior a la de los médicos.

5. Sería una alternativa al problema insoluble de la oferta de médicos y se lograría una mejor utilización de los disponibles.

6. Se darían nuevas oportunidades de empleo a las personas que no alcanzan estudios universitarios.

En varios paises del mundo existe amplia experiencia en este tipo de sistemas, que podría ser aprovechada para una mejor implantación del mismo en México.

\section{CONCLUSIONES}

No hay ninguna duda con respecto a la existencia de un problema de escasez actual y futura de médicos para satisfacer la demanda, aun definida en los términos restringidos de Myers. Sin embargo, nos parece que la misma falta de oferta respecto a la demanda efectiva (que depende del ingreso) - de la que queda fuera por lo menos un $60 \%$ de la población- tiende a satisfacerse a través de los mecanismos del mercado. Un ejemplo muy claro es la tendencia al establecimiento de escuelas de medicina de parte de la iniciativa privada que, dadas las circunstancias en que se crean (personal docente a tiempo completo, hospitales universitarios, etc.) probablemente producirán médicos de mayor calidad en el país. Como consecuencia, es de esperar que los egresados de estas escuelas tenderán a satisfacer las demandas de servicios médicos de los sectores más acomodados o bien éstos seguirán haciendo uso de los servicios médicos del extranjero. ${ }^{6}$

El problema grave y que nos parece Myers deja fuera de sus ecuaciones es la demanda de servicios médicos de los demás sectores de la población. Por ello es imprescindible un replanteamiento total del problema, dada la dificultad de resolverlo con los esquemas actuales.

Creemos que en el caso de la demanda de servicios de salud pública, juegan un papel tan importante las decisiones de política que se hace casi imposible prever lo que sucederá en el futuro.

La decisión de la presente administración de llevar el seguro social al campo, sin un cambio en la forma de proveer los servicios, nos hace pensar que habrá un cambio notable en la estructura de la oferta de servicios médicos así como en la demanda de ellos. El problema en los términos planteados por Myers se verá agudizado.

6 Se estima que el gasto anual en servicios médicos del exterior es de $600 \mathrm{mi}$ llones de pesos. 